\title{
A Survey of Coping Strategies With Stress in Patients With Acute Myocardial Infarction and Individuals Without a History of Fixed Myocardial Infarction
}

\author{
Seyed Mahmood Sadr Bafghi ${ }^{a}$, Nastaran Ahmadia, e, Seyyed Mojtaba Yassini Ardekani ${ }^{\text {b }}$ \\ Lida Jafaric ${ }^{\mathrm{c}}$, Bahareh Bitaraf Ardekani ${ }^{\mathrm{d}}$, Roya Heydaric, \\ Fahame Maroufic, Reza Faraji ${ }^{\mathrm{a}}$
}

\begin{abstract}
Background: This study aimed at investigating the coping strategies with stress in patients with acute myocardial infarction (MI) and individuals without a history of fixed MI and cardiovascular disorders.

Methods: This case-control crossover study was conducted from March 2015 to February 2016 on 220 patients with acute MI (MI patients) as case group and 220 patients without any history of MI and cardiovascular diseases as the control group using availability sampling method. To collect the required data, demographic information questionnaire, Holms-Raheh life stress inventory, perceived stress questionnaire, and coping inventory for stressful situations (CISS) were applied.

Results: On the basis of our findings, 118 patients (53.6\%) with MI used emotion-focused coping strategy. Ninety-seven patients $(82.2 \%)$ with MI who used emotion-focused coping strategy had negative perceived stress. Additionally, 71 patients $(60.2 \%)$ with MI who had used emotion-focused coping strategy suffered from very high level of stress.

Conclusion: The most MI patients had very high level of stress while most people in control group had high level of stress. Most MI patients that had very high level of stress cope with it in emotion-focused coping strategy and it proves that people with higher levels of stress are more likely to use inefficient coping strategies.
\end{abstract}

Keywords: Stress; Acute myocardial infarction; Coping strategies

Manuscript submitted December 11, 2017, accepted December 27, 2017

aYazd Cardiovascular Research Center, Shahid Sadoughi University of Medical Sciences, Yazd, Iran

${ }^{b}$ Department of Psychiatry, School of Medicine, Shahid Sadoughi University of Medical Sciences, Yazd, Iran

'Department of Clinical Psychology, Yazd Branch, Islamic Azad University, Yazd, Iran

${ }^{\mathrm{d} C o u n s e l i n g ~ P s y c h o l o g y, ~ A s h k e z a r ~ B r a n c h, ~ I s l a m i c ~ A z a d ~ U n i v e r s i t y, ~}$ Ashkezar, Iran

${ }^{\mathrm{e}}$ Corresponding Author: Nastaran Ahmadi, Afshar Hospital, Jomhori Blv, Yazd, Iran. Email: ahmadi.psy@gmail.com

doi: https://doi.org/10.14740/cr655w with stress

\section{Introduction}

Cardiovascular diseases are considered as one of the three main causes of mortality along with cancer and stroke in the developed countries [1-3]. Today, the prevalence of cardiovascular diseases is increasing constantly so that according to WHO, it is anticipated that the prevalence rate of the disorder will reach $46.4 \%$ by 2020 [2]. It is estimated that 1.5 million cases of myocardial infarction (MI) will be identified annually in the USA [4]. The complications of this disorder not only affect the patients' health, but also influence the social relations, communications, familial atmosphere, and occupation imposing unexpected costs on the patients $[5,6]$. Coronary artery disease (CAD) has been rendered as the most fatal of cardiovascular disorders. It emerges as the consequence of coronary artery stenosis or obstruction [7]. Various factors contribute to this disease increasing the risk of affliction with it. Among these risk factors mention can be made of diabetes, hyperlipidemia, obesity, lack of regular physical activity and sedentary living, gender, age, family history, and psychological factors like stress [8-14]. Of these, stress can play a role as a significant parameter in the incidence of acute MI [15, 16], though some studies have not mentioned stress as one of the main causes of cardiovascular diseases [17]. Stress is defined as the physical, mental, and emotional responses experienced due to changes in individual's life and needs of the personal life [18]. It has been demonstrated in the coronary disease patients that the stress level in these patients is higher than that of the healthy individuals in the community [19]. Lazarus et al have defined coping strategies as a collection of behavioral and cognitive responses aimed at minimizing the pressure of stressful life situations [20]. Stress coping strategies may be helpful in cardiovascular patients. Williams et al suggested in their study that educating cardiovascular patients in stress coping strategies was associated with reduced stress in these patients [21]. Hence, regarding the increasing incidence of MI and the health researchers' growing emphasis on the correlation between psycho-social factors and incidence and continuation of car- 
Table 1. Comparison of Coping Strategies in Patients With Acute Myocardial Infarction and Individuals Without a History of Fixed Myocardial Infarction

\begin{tabular}{lllll}
\hline \multirow{2}{*}{ Group } & \multicolumn{3}{c}{ Coping strategies } & \\
\cline { 2 - 5 } & Problem focused & Emotion focused & Avoidance behavior & Total \\
\hline MI, no. (\%) & $74(33.6 \%)$ & $118(53.6 \%)$ & $28(12.7 \%)$ & 220 \\
Control, no. (\%) & $140(63.6 \%)$ & $13(5.9 \%)$ & $67(30.4 \%)$ & 220 \\
Total, no. (\%) & $214(48.6 \%)$ & $131(29.7 \%)$ & $95(21.5 \%)$ & 440 \\
\hline
\end{tabular}

diovascular diseases [22, 23], the necessity of teaching stress coping strategies in these patients is felt more than ever.

\section{Materials and Methods}

This case-control crossover study was carried out for 1 year in Afshar Hospital in Yazd, central Iran, from March 2015 to February 2016. The study population included 220 patients with MI assigned to the case group and 220 individuals without MI or cardiovascular diseases who participated in Yazd healthy heart project and YAHS project, assigned to the control group. These individuals who participated in the study were selected using the availability sampling method. The inclusion criteria were: affliction with acute MI, diagnosis of $\mathrm{MI}$ according to WHO criteria by the cardiologist, being awake, and sufficient cooperation with the psychologist. Also, the exclusion criteria were: affliction with psychological disorders over the last 6 months. After definite diagnosis and the patient's relative recovery and getting rid of medical emergency state, the demographic information questionnaire, the Holms-RaheLife stress inventory, the perceived stress questionnaire, and the coping inventory for stressful situations (CISS) were completed for the patients by psychologist and the required data were gleaned.

\section{Holmz-Rahe life stress inventory}

This instrument is designed for assessing 43 life-altering events by Thomas Holms and Richard Drahe in 1967. These events are called series of recent events (SREs). On the basis of Holms-Rahe scale, life alterations over the last 2 years are assessed and the total score is calculated. If the total score ranges between 150 and 200, there is a 37\% odds of affliction with a disease during the year. If the total score ranges between 200 and 300 or more than 300, there is a probability of $50 \%$ and $80 \%$ of affliction with the disease, respectively. In this study, the patients' ranks were considered as less than 150, $151-200$, and $201-300$ on the basis of score of life events [24].

\section{The perceived stress questionnaire}

The perceived stress scale was developed by Cohen et al (1983) and includes three versions: the four-item, the 10-item, and the 14-item versions which are used to assess the gen- eral perceived stress over the last month. It measures the feelings and thoughts about stressful events, control, overcoming and success, withstanding the psychological stress, and the experienced stresses. Moreover, this scale investigates the risk factors of behavioral disorders and displays the stressful relations. A higher score indicates a greater perceived stress. In the present study, Cronbach's alpha correlation coefficient was 0.74 for this tool. To establish the criterion-referenced validity of this scale, Cohen et al (1983) estimated its correlation coefficient between 0.52 and 0.76 using symptomatological measures [25]. The inventory was scored by a five-point Likert scale ranging from never $(=0)$, almost never $(=1)$, sometimes $(=2)$, often $(=3)$, to very often $(=4)$. It includes two subscales: 1) the negative perceived stress subscale which includes items $1,2,3,8,11,12$, and 14, and 2) the positive perceived stress subscale which includes items 4, 5, 6, 7, 9, 10 , and 13 . Items $4,5,6,7,9,10$ and 13 are scored conversely and include never $(=4)$ to very often $(=0)$. The minimum obtained score is 0 , while the maximum score is 56 points. The cut-off score is 21.8 and a higher score indicates negative perceived stress.

\section{CISS}

This instrument was developed by Andler and Parker (1990). This test contains 48 items in which the responses are marked as never $(=1)$ to always $(=5)$. The CISS includes the three main fields of coping behaviors: 1) problem-focused coping strategy or active approach to the problem management and solution; 2) emotion-focused coping strategy or concentration on emotional responses to the problem; and 3) avoidance behavior strategy or escaping the problem. Given that this tool is in the five-point Likert scale format, the maximum score for each item is 5 points while the minimum score is 1 . The respondents should reply to all the items. If the subject does not respond to five items or less, the researcher can mark choice 3 of the incomplete items, otherwise, the questionnaire is not scored, i.e., when more than five items are not marked. The range of changes in the three coping behaviors is in a way that the score of each of the coping behaviors, i.e., problemfocused, emotion-focused, and avoidance behavior ranges between 16 and 80 . In other words, the individual's dominant coping method is determined by the score obtained in the test. This means that the behavior with a higher score is taken as individual's stress coping behavior [26]. This study was approved by the Committee of Ethics in Medical Research at the Shahid Sadoughi University of Medical Sciences, Yazd, 
Table 2. Comparison of Coping Strategies on the Basis of Perceived Stress in Patients With Acute Myocardial Infarction and Individuals Without a History of Fixed Myocardial Infarction

\begin{tabular}{|c|c|c|c|}
\hline \multirow{2}{*}{ Group } & \multicolumn{2}{|c|}{ Perceived stress } & \multirow{2}{*}{ Total } \\
\hline & Positive & Negative & \\
\hline \multicolumn{4}{|l|}{ MI, no. (\%) } \\
\hline Emotion focused & $21(17.8 \%)$ & $97(82.2 \%)$ & $118(100.0 \%)$ \\
\hline Avoidance behavior & $0(0.0 \%)$ & $28(100.0 \%)$ & $28(100.0 \%)$ \\
\hline Problem focused & $39(27.9 \%)$ & $101(72.1 \%)$ & $140(100.0 \%)$ \\
\hline Emotion focused & $0(0.0 \%)$ & $13(100.0 \%)$ & $13(100.0 \%)$ \\
\hline Avoidance behavior & $35(52.2 \%)$ & $32(47.8 \%)$ & $67(100.0 \%)$ \\
\hline Total & $74(33.6 \%)$ & $146(66.4 \%)$ & $220(100.0 \%)$ \\
\hline
\end{tabular}

Iran.

\section{Results}

This case-control crossover study was conducted over 1 year from March 2015 to February 2016 on 220 MI patients as the case group and 220 individuals without a history of MI or cardiovascular diseases as the control group. The participants in the case group were $149(68.3 \%)$ males and $71(31.7 \%)$ females. Also, there were $100(45.5 \%)$ males and $120(54.5 \%)$ females in the control group. On the basis of our findings, 118 $(53.6 \%)$ MI patients used the emotion-focused coping strategy while in the control group, $140(63.6 \%)$ individuals applied the problem-focused coping strategy (Table 1). Moreover, $97(82.2 \%)$ MI patients, who used the emotion-focused coping strategy, had negative perceived stress. Additionally, $101(72.1 \%)$ individuals in the control group who made use of problem-focused coping strategy showed negative perceived stress (Table 2). A comparison of the results of stress coping strategies on the basis of stress level in both groups demonstrated that $71(60.2 \%)$ MI patients who used emotion-focused coping strategy suffered from very high level of stress. Furthermore, 75 (53.6\%) individuals who used problem-focused coping strategy sustained high level of stress (Table 3 ).

\section{Discussion}

The aim of the present study was to investigate the coping strategies with stress in patients with acute MI and individuals without a history of fixed MI and cardiovascular disorders. In this study, coping strategies on the basis of perceived stress and stress level also was measured. We found that most MI patients used emotion-focused coping strategy. Previous studies showed inconsistent findings in this regard. In a study, Chung et al investigated that MI patients used problem-focused more than emotion-focused types of coping and this difference is probably due to that in Chung's study, their samples were less than our study and the onset of their MI who participated in Chung's study was

Table 3. Comparison of Coping Strategies on the Basis of Stress Level in Patients With Acute Myocardial Infarction and Individuals Without a History of Fixed Myocardial Infarction

\begin{tabular}{|c|c|c|c|c|c|}
\hline \multirow{2}{*}{ Group } & \multicolumn{4}{|c|}{ Stress level } & \multirow{2}{*}{ Total } \\
\hline & Low & Moderate & High & Very high & \\
\hline \multicolumn{6}{|l|}{ MI, no. (\%) } \\
\hline Emotion focused & $22(18.6 \%)$ & $4(3.4 \%)$ & $21(17.8 \%)$ & $71(60.2 \%)$ & $118(100.0 \%)$ \\
\hline Avoidance behavior & $2(7.1 \%)$ & $6(21.4 \%)$ & $5(17.9 \%)$ & $15(53.6 \%)$ & $28(100.0 \%)$ \\
\hline Problem focused & $18(12.9 \%)$ & $11(7.9 \%)$ & $75(53.6 \%)$ & $36(25.7 \%)$ & $140(100.0 \%)$ \\
\hline Emotion focused & $2(15.4 \%)$ & $4(30.8 \%)$ & $5(38.5 \%)$ & $2(15.4 \%)$ & $13(100.0 \%)$ \\
\hline Avoidance behavior & $13(19.4 \%)$ & $3(4.5 \%)$ & $26(38.8 \%)$ & $25(37.3 \%)$ & $67(100.0 \%)$ \\
\hline Total & $33(15.0 \%)$ & $18(8.2 \%)$ & $106(48.2 \%)$ & $63(28.6 \%)$ & $220(100.0 \%)$ \\
\hline
\end{tabular}


less than 1 month prior to the study [27]. Emotion-focused coping strategies are used instead of focusing on the actual situation of the problem such as: suppressing (stopping/inhibition of) negative thoughts or emotions, praying, eating more, drinking alcohol, using drugs, releasing pent-up emotions, distracting oneself, meditating, blame, disclaiming, seeking social support. This strategy is very useful in dealing with situations that cannot be changed and when one needs to accept reality and can be both positive and negative coping methods. However, most participants in the control group used problem-focused coping strategy. Problem-focused coping strategy finds out information on the problem and learns new skills to manage the problem and try to deal with the cause of their problem. It has three techniques: taking control, information seeking, and evaluating the pros and cons. It includes: problem-solving, time-management, and obtaining instrumental social support.

Since coping strategies were a partial mediator between different levels of stress and acute MI, our findings are consistent with studies showing the level of stress and coping strategies are related [28-31]. However, little is known about the relationship between psychological distress and coping strategies in MI patients. This study demonstrated that most MI patients had very high level of stress while most people in control group had high level of stress. This result shows that higher stress over the past 2 years can be one of the causes of MI. Also the results showed that most MI patients that had very high level of stress cope with it in emotion-focused coping strategy and it proves that people with higher levels of stress are more likely to use inefficient coping strategies.

Our results basically suggest that most participants in two groups appraise most situations in their life as stressful. Also, according to our results most MI patients that find their lives in the form of unpredictable, uncontrollable, and overloaded respondents (negative perceived stress) cope with their stress in an emotion-focused coping strategy. Some studies have also argued that the coping strategy is a significant moderating variable that affects the level of negative perception of stress when an individual exposed the stressor $[32,33]$.

This study establishes case-control associations, and further study is necessary to identify causal relationships between the levels of stress, perceived stress and coping strategies with stress in MI patients.

\section{Conclusion}

It is important to assess coping strategies for MI patients and to provide interventions to use more positive coping strategies like problem-focused coping strategy. Problem-focused coping strategy can help patients to reduce the likelihood of another MI occurring. Also, if we can change the patient's view about perception of stress, then we can change their process of cognition about stressful situation and finally change their lifestyle.

\section{Acknowledgments}

The authors of this article give their special thanks to Yazd
Cardiovascular Research Center, and Shahid Sadoughi University of Medical Sciences, Yazd, Iran.

\section{Competing Interests}

The authors declare that they have no competing interests.

\section{Grant Support}

The study was supported by the Shahid Sadoughi University of Medical Sciences (No IR.SSU.REC.1394.111).

\section{Financial Disclosure}

None.

\section{References}

1. Kasper DL, Braunwald E, Fauci AS, Hauser SL, Longo DL, Jameson JL, Harrison S. Principles of internal medicine. New York. McGrow-Hill; 2005.

2. Reynolds S, Waterhouse K, Miller KH. Head of bed elevation, early walking, and patient comfort after percutaneous transluminal coronary angioplasty. Dimens Crit Care Nurs. 2001;20(3):44-51.

3. Leonardi M, Raggi A, Pagani M, Carella F, Soliveri P, Albanese A, Romito L. Relationships between disability, quality of life and prevalence of nonmotor symptoms in Parkinson's disease. Parkinsonism Relat Disord. 2012;18(1):35-39.

4. MacInnes JD. The illness perceptions of women following acute myocardial infarction: implications for behaviour change and attendance at cardiac rehabilitation. Women Health. 2005;42(4):105-121.

5. Byrne M, Walsh J, Murphy AW. Secondary prevention of coronary heart disease: patient beliefs and health-related behaviour. J Psychosom Res. 2005;58(5):403-415.

6. Bajenaru O, Antochi F, Tiu C. Particular aspects in patients with coronary heart disease and vascular cognitive impairment. J Neurol Sci. 2010;299(1-2):49-50.

7. Takemura M, Matsumoto H, Niimi A, Ueda T, Matsuoka $\mathrm{H}$, Yamaguchi M, Jinnai M, et al. High sensitivity C-reactive protein in asthma. Eur Respir J. 2006;27(5):908-912.

8. Jiang W, Krishnan RR, O'Connor CM. Depression and heart disease: evidence of a link, and its therapeutic implications. CNS Drugs. 2002;16(2):111-127.

9. Lett HS, Blumenthal JA, Babyak MA, Sherwood A, Strauman T, Robins C, Newman MF. Depression as a risk factor for coronary artery disease: evidence, mechanisms, and treatment. Psychosom Med. 2004;66(3):305-315.

10. Rugulies R. Depression as a predictor for coronary heart disease. a review and meta-analysis. Am J Prev Med. 2002;23(1):51-61.

11. Perers E, Caidahl K, Herlitz J, Karlsson T, Hartford M. 
Impact of diagnosis and sex on long-term prognosis in acute coronary syndromes. Am Heart J. 2007;154(3):482488.

12. Ho PM, Luther SA, Masoudi FA, Gupta I, Lowy E, Maynard C, Sales AE, et al. Inpatient and follow-up cardiology care and mortality for acute coronary syndrome patients in the Veterans Health Administration. Am Heart J. 2007;154(3):489-494.

13. Lippi G, Targher G, Montagnana M, Salvagno GL, Guidi GC. Relationship between gamma-glutamyltransferase, lipids and lipoprotein(a) in the general population. Clin Chim Acta. 2007;384(1-2):163-166.

14. Braunwald E. Heart disease: a text book of cardiovascular medicine. 9th ed. Philadelphia: Saunders/Elsevier; 2012.

15. Kinjo K, Sato H, Shiotani I, Kurotobi T, Ohnishi Y, Hishida E, Nakatani D, et al. Variation during the week in the incidence of acute myocardial infarction: increased risk for Japanese women on Saturdays. Heart. 2003;89(4):398403.

16. Gullette EC, Blumenthal JA, Babyak M, Jiang W, Waugh RA, Frid DJ, O'Connor CM, et al. Effects of mental stress on myocardial ischemia during daily life. JAMA. 1997;277(19):1521-1526.

17. Woods SL, Froelicher ES, Motzer SA, Bridges EJ. Cardiac nursing; 5th edition. Philadelphia: Lippincott Williams \& Wilkins; 2005.

18. Soulas T, Sultan S, Gurruchaga JM, Palfi S, Fenelon G. Changes in quality of life, burden and mood among spouses of Parkinson's disease patients receiving neurostimulation. Parkinsonism Relat Disord. 2012;18(5):602-605.

19. Hallman T, Thomsson H, Burell G, Lisspers J, Setterlind S. Stress, burnout and coping: differences between women with coronary heart disease and healthy matched women. J Health Psychol. 2003;8(4):433-445.

20. Lazarus RS, Folkman S. Stress, appraisal and coping. New York: Springer:1984.

21. Williams RB, Barefoot JC, Schneiderman N. Psychosocial risk factors for cardiovascular disease: more than one culprit at work. JAMA. 2003;290(16):2190-2192.

22. Smith TW, Ruiz JM. Psychosocial influences on the de- velopment and course of coronary heart disease: current status and implications for research and practice. J Consult Clin Psychol. 2002;70(3):548-568.

23. Sadock B, Sadock V. Kaplan and Sadock's comprehensive textbooks of psychiatry. 8th ed. New York : Williams Wilkins, 1998.

24. Holms TH, Rahe RH. The social readjustment rating scale. J Psycho Res. 1967;11:213-218.

25. Ramirez MT, Hernandez RL. Factor structure of the Perceived Stress Scale (PSS) in a sample from Mexico. Span J Psychol. 2007;10(1):199-206.

26. Cosway R, Endler N, Sadler AJ, Deary LJ. The coping inventory for stressful situations: factoria structure and associations with personality traits and psychological health. J Appl Biobehav Res. 2007;5:121-143.

27. Chung MC, Dennis I, Berger Z, Jones R, Rudd H. Posttraumatic stress disorder following myocardial infarction: personality, coping, and trauma exposure characteristics. Int J Psychiatry Med. 2011;42(4):393-419.

28. Son H, Thomas SA, Friedmann E. The association between psychological distress and coping patterns in postMI patients and their partners. J Clin Nurs. 2012;21(1516):2392-2394.

29. Kanninen K, Punamaki RL, Qouta S. The relation of appraisal, coping efforts, and acuteness of trauma to PTS symptoms among former political prisoners. J Trauma Stress. 2002;15(3):245-253.

30. Solomon Z, Avitzur E, Mikulincer M. Coping resources and social functioning following combat stress reaction: A longitudinal study. J Soc Clin Psychol. 1989;8:87-96.

31. Gil S. Coping style in predicting posttraumatic stress disorder among Israeli students. Anxiety. Stress Coping. 2005;18:351-359.

32. Fuller BF, Conner DA. Selection of vigilant and avoidant coping strategies among repressors, highly anxious and truly low anxious subjects. Psychol Rep. 1990;66(1):103110.

33. Miller SM. Coping with impending stress: psychophysiological and cognitive correlates of choice. Psychophysiology. 1979;16(6):572-581. 